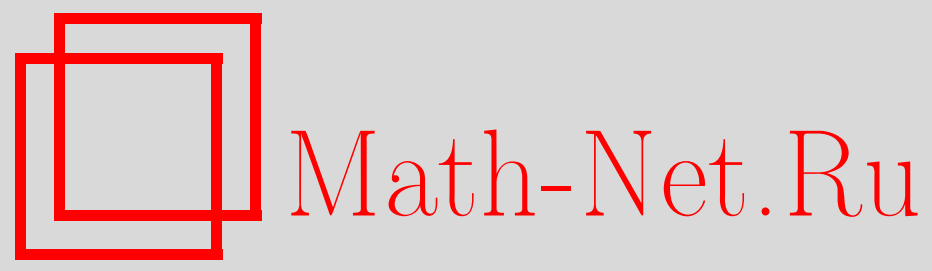

Е. Н. Огородников, Об одном классе дробных дифференциальных уравнений математических моделей динамических систем с памятью, Вестн. Сам. гос. техн. ун-та. Сер. Физ.-мат. науки, 2013, выпуск 1(), 245252

DOI: https://doi.org/10.14498/vsgtu1224

Использование Общероссийского математического портала Math-Net.Ru подразумевает, что вы прочитали и согласны с пользовательским соглашением

http: //www.mathnet.ru/rus/agreement

Параметры загрузки:

IP : 3.91 .87 .62

26 апреля 2023 г., 13:44:59

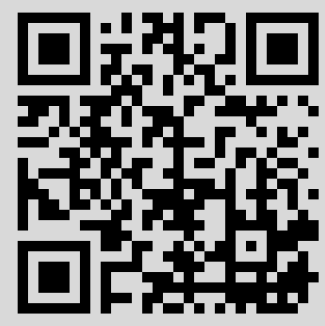




\title{
ОБ ОДНОМ КЛАССЕ ДРОБНЫХ ДИФФЕРЕНЦИАЛЬНЫХ УРАВНЕНИЙ МАТЕМАТИЧЕСКИХ МОДЕЛЕЙ ДИНАМИЧЕСКИХ СИСТЕМ С ПАМЯТЬЮ
}

\author{
Е. Н. Огородников \\ Самарский государственный технический университет, \\ Россия, 443100, Самара, ул. Молодогвардейская, 244. \\ E-mail: eugen.ogo@gmail.com
}

\begin{abstract}
Рассмотрено дифференииалъное уравнение с дробными производными РиманаЛиувилля, которое предлагается в качестве моделъного дробно-осииллячионного уравнения для описания колебательных процессов в динамических системах с памятью. В основе его вывода лежит гипотеза о неидеальной вязкоупругой связи, которая ассоциируется с дробным аналогом реологической модели Зенера, представляющей собой в классическом случае параллельное соединение элемента Максвелла и идеальной пружины. Показано, что начальные задачи типа Коши эквивалентным образом редуцируются $\kappa$ интегралъным уравнениям волътерровского типа с достаточно гладкими ядрами,что позволяет воспользоватъся методом последовательных приближений. Отмечено, что подобные дифббереницальные уравнения могут представлять интерес в качестве математических моделей поведения нелинейных динамических систем.
\end{abstract}

Ключевые слова: дифберенциалъные и интегралънъе уравнения с дробными операторами Римана-Лиувилля, дробнье осиилляторь, дробно-осциляционные уравнения, реологические модели вязкоупругого тела с памятъю, специальнье бункции типа Миттаг-Леффлера, интегральные уравнения Вольтерры со спеицальными функциями в ядрах.

Дифференциальное уравнение

$$
\ddot{u}+\sum_{k=0}^{n} a_{k} D_{0 t}^{\alpha_{k}} \dot{u}+\sum_{s=0}^{m} b_{s} D_{0 t}^{\beta_{s}} u=f(t),
$$

где $u(t)$ - искомая, а $f(t)$ - заданная функции, $t \in[0, T], \dot{u}=d u / d t, D_{0 t}^{\alpha} u=$ $=\left(D_{0+}^{\alpha} u\right)(t)$ - левосторонняя дробная производная Римана-Лиувилля $[1,2]$ порядка $\alpha, \alpha_{k} \in(0,1), \beta_{s} \in[0,2)$, а коэффициенты $a_{k}, b_{s} \in \mathbb{R}$, представляет собой обобщение дифференциального уравнения классического осциллятора с вязким трением [3]. Некоторые простейшие математические модели дробных осцилляторов приведены в $[2,4,5]$.

Уравнению (1), различным его частным случаям, вопросам обоснования существования и единственности и структуре решения начальных задач был посвящён ряд работ с участием автора данного сообщения [3, 6, 7 и др.].

В основе вывода уравнения (1) лежит гипотеза о неидеальной вязкоупругой связи, которая ассоциируется с дробным аналогом обобщённой реологической модели Фойхта

$$
\sigma(t)=\sum_{k=0}^{n} a_{k} D_{0 t}^{\alpha_{k}} \dot{\varepsilon}+\sum_{s=0}^{m} b_{s} D_{0 t}^{\beta_{s}} \varepsilon,
$$

Евгений Николаевич Огородников (к.ф.-м.н.), доцент, каф. прикладной математики и информатики. 
где $\sigma=\sigma(t)$ и $\varepsilon=\varepsilon(t)$ - напряжение и деформация связи в момент времени $t$. Частные случаи определяющего соотношения (2) изучались в работе [8] и были использованы авторами работы [7] в простейших модельных уравнениях дробных осцилляторов. Однако соотношение (2) ещё нуждается в экспериментальном подтверждении. В то время как экспериментально установлено [9], что для моделирования вязкоупругого поведения многих материалов достаточно адекватным оказывается дробный аналог реологической модели Кельвина

$$
\sigma(t)+a_{1} D_{0 t}^{\alpha} \sigma=E_{0} \varepsilon(t)+b_{1} D_{0 t}^{\alpha} \varepsilon,
$$

где $a_{1}, b_{1}, E_{0}$ - заданные постоянные величины, $\alpha \in(0,1)$.

Отметим, что определяющее соотношение вида (3) предлагалось и другими авторами [10]. Однако большинство авторов используют в реологических соотношениях «подходящую дробную производную», в качестве которой обычно выбирается производная по Капуто [5].

Другим подтверждённым экспериментально реологическим соотношением является дробный аналог модели Максвелла

$$
\sigma(t)+a_{1} D_{0 t}^{\alpha} \sigma=b_{1} D_{0 t}^{\alpha} \varepsilon, \quad \alpha \in(0,1) .
$$

Известно [2], что к соотношению (4) редуцируется определяющее уравнение Ю. Н. Работнова [11].

В настоящей работе в качестве определяющего соотношения, моделирующего вязкоупругую связь, предложен дробный аналог модели Зенера, представляющей собой в классическом случае параллельное соединение максвелловского элемента и идеальной пружины. Заменяя в структурной модели Зенера элемент Максвелла (последовательное соединение пружины и демпфера) его дробным аналогом (4), приходим к соотношению

$$
\sigma(t)+a_{1} D_{0 t}^{\alpha} \sigma=E \varepsilon(t)+\left(a_{1} E+b_{1}\right) D_{0 t}^{\alpha} \varepsilon,
$$

содержащему четыре параметра: $a_{1}, b_{1}, E, \alpha$, подлежащих идентификации по результатам экспериментов (испытаний образца). Предполагается, что при $\alpha=1$ соотношение (5) совпадает с классическим для модели Зенера соотношением

$$
\sigma(t)+a_{1} \dot{\sigma}(t)=E_{2}\left(\varepsilon(t)+a_{1} \dot{\varepsilon}(t)\right)+\eta \dot{\varepsilon}(t),
$$

где $a_{1}=\eta / E_{1} ; E_{1}, E_{2}$ - модули упругости, $\eta-$ коэффициент вязкости соответствующих конструкционных элементов модели Зенера.

Дифференциальное уравнение динамики частицы (материальной точки) в рамках классической механики Ньютона при наличии неидеальной вязкоупругой связи (5) и внешнего воздействия (активной силы) $f(t)$ будет иметь вид

$$
\left(D_{0 t}^{\alpha}-\lambda I\right)\left(m \ddot{x}+c_{2} x-f\right)+c_{1} D_{0 t}^{\alpha} x=0,
$$

где $m$ - масса, а $x=x(t)$ - координата частицы; $\lambda, c_{1}, c_{2}$ - некоторые константы, связанные с коэффициентами в равенстве $(5) ; I$ - тождественный оператор, $D_{0 t}^{\alpha}-$ левосторонняя производная Римана-Лиувилля порядка $\alpha \in$ $(0,1)$. 
Введём функции $u(t)=m \ddot{x}+c_{2} x-f$ и $v(t)=-c_{1} D_{0 t}^{\alpha} x$. Если считать $v(t)$ известной функцией, то уравнение (6) является неоднородным дифференциальным уравнением Барретта относительно искомой функции $u(t)[12]$ :

$$
D_{0 t}^{\alpha} u-\lambda u(t)=v(t)
$$

для которого корректно поставлена задача типа Коши с начальным условием

$$
\lim _{t \rightarrow 0+} I_{0 t}^{1-\alpha} u=u_{0}
$$

Будем искать решение $x(t)$ дифференциального уравнения (6) в классе функций $A C^{2}[0, T]$, допускающем существование почти всюду на отрезке $[0, T]$ суммируемой второй производной $\ddot{x}(t)[1]$, требуя кроме того, чтобы $D_{0 t}^{\alpha} \ddot{x}$ была суммируемой по Лебегу функцией. Но это значит, что искомое решение $x(t)$ дифференциального уравнения (6) должно быть таким, что $\ddot{x}(t) \in L^{\alpha}(0, T)$, где класс функций $L^{\alpha}(0, T)$ при $\alpha \in(0,1)$ мы определяем как множество функций $\varphi(t)$ таких, что $\varphi(t) \in L(0, T)$, а $I_{0 t}^{1-\alpha} \in A C^{1}[0, T]$ [13], где $A C^{1}[0, T]=A C[0, T]$ - класс абсолютно непрерывных функций. Напомним, что класс функций $A C^{n}[a, b](n \in \mathbb{N})$ определяется как множество функций $\varphi(x)(x \in[a, b])$ таких, что $\varphi(x) \in C^{n-1}[a, b]$, a $f^{(n-1)}(x) \in A C[a, b]$, что гарантирует существование почти всюду на $[a, b]$ суммируемой производной $f^{(n)}(x)$.

Таким образом, в указанном классе функций начальное условие (8) для внешнего возмущения $f(t) \in C[0, T]$ фактически сводится к равенству

$$
\lim _{t \rightarrow 0+} I_{0 t}^{1-\alpha} \ddot{x}=u_{0}
$$

Применяя к левой и правой частям равенства $(7)$ оператор $I_{0 t}^{\alpha}$ и используя известное тождество [1]

$$
I_{0 t}^{\alpha} D_{0 t}^{\alpha} u=u(t)-\frac{t^{\alpha-1}}{\Gamma(\alpha)} \lim _{t \rightarrow 0+} I_{0 t}^{1-\alpha} u,
$$

справедливое для функций $u(t) \in L^{\alpha}(0, T)$, где $\Gamma(\alpha)$ - гамма-функция Эйлера, получим интегральное уравнение

$$
u(t)-\lambda I_{0 t}^{\alpha} u=I_{0 t}^{\alpha} v
$$

Его решение с учётом того, что $I_{0 t}^{\alpha} v=-c_{1} I_{0 t}^{\alpha} D_{0 t}^{\alpha} x=-c_{1} x(t)$, для функций $x(t) \in A C[0, T]$ и, тем более, для функций $x(t) \in A C^{2}[0, T]$, легко записывается с помощью оператора $\left(I-\lambda I_{0 t}^{\alpha}\right)^{-1}=I+\lambda E_{0 t ; \lambda}^{\alpha, \alpha}$ :

$$
u(t)=\left(I+\lambda E_{0 t ; \lambda}^{\alpha, \alpha}\right)\left(\frac{u_{0}}{\Gamma(\alpha)} t^{\alpha-1}-c_{1} x(t)\right),
$$

где действие оператора $E_{a t}^{\alpha, \sigma}$ на любую суммируемую функцию определяется равенством

$$
E_{a t ; \lambda}^{\alpha, \sigma} f=\int_{a}^{t}(t-\tau)^{\alpha-1} E_{\sigma}\left[\lambda(t-\tau)^{\alpha} ; \alpha\right] f(\tau) d \tau
$$


где $E_{\alpha}(z ; \mu)$ - функция типа Миттаг-Леффлера. ности,

Некоторые свойства операторов $E_{a t ; \lambda}^{\alpha, \sigma}$ и $I+\lambda E_{a t ; \lambda}^{\alpha, \alpha}$ приведены в $[7,8]$. В част-

$$
\left(I+\lambda E_{0 t ; \lambda}^{\alpha, \alpha}\right) t^{\alpha-1}=\Gamma(\alpha) \operatorname{Exp}(\alpha, \alpha ; \lambda ; t),
$$

где $E(\alpha, \mu ; \lambda ; t)=t^{\mu-1} E_{\alpha}\left(\lambda t^{\alpha} ; \mu\right)$ - обобщённая дробная экспоненциальная функция [6].

Таким образом, решение дифференциального уравнения (6) с начальным условием (8) или (9) редуцировано к интегро-дифференциальному уравнению следующего вида:

$$
m \ddot{x}+\left(c_{1}+c_{2}\right) x+c_{1} \lambda E_{0 t ; \lambda}^{\alpha, \alpha} x=f(t)+u_{0} \operatorname{Exp}(\alpha, \alpha ; \lambda ; t) .
$$

Обозначим $\omega_{1}^{2}=c_{1} / m, \omega_{2}^{2}=c_{2} / m, \omega_{0}^{2}=\omega_{1}^{2}+\omega_{2}^{2}$ и $f_{1}(t)=m^{-1} f(t)$. Дальнейшая редукция уравнения (11) к интегральному уравнению второго рода может быть осуществлена двумя способами.

Запишем уравнение (11) в виде

$$
\ddot{x}+\omega_{0}^{2} x+\omega_{1}^{2} \lambda E_{0 t ; \lambda}^{\alpha, \alpha} x=f_{1}(t)+\frac{u_{0}}{m} \operatorname{Exp}(\alpha, \alpha ; \lambda ; t) .
$$

Интегрируя левую и правую части равенства (12) по $t$ дважды с начальными условиями

$$
x(0)=x_{0}, \quad \dot{x}(0)=\dot{x}_{0}
$$

и используя известные свойства функции $\operatorname{Exp}(\alpha, \mu ; \lambda ; t)$ и оператора $E_{0 t ; \lambda}^{\alpha, \sigma}$, приводим уравнение (12) к интегральному уравнению Вольтерры второго рода

$$
x(t)+\left(\omega_{1}^{2} E_{0 t ; \lambda}^{2, \alpha}+\omega_{2}^{2} I_{0 t}^{2}\right) x(t)=x_{0}+\dot{x}_{0} t+\frac{u_{0}}{m} \operatorname{Exp}(\alpha, \alpha+2 ; \lambda ; t)+I_{0 t}^{2} f_{1} .
$$

Нетрудно показать, что ядро интегрального оператора

$$
\left(\omega_{1}^{2} E_{0 t ; \lambda}^{2, \alpha}+\omega_{2}^{2} I_{0 t}^{2}\right) x(t)=\int_{0}^{t}\left[\omega_{1}^{2} \operatorname{Exp}(\alpha, 2 ; \lambda ;(t-\tau))+\omega_{2}^{2}(t-\tau)\right] x(\tau) d \tau
$$

принадлежит классу функций $C^{1}[0, T] \cap C^{2}(0, T)$, а правая часть интегрального уравнения (14) - классу функций $C^{2}[0, T]$. Более того, обозначая ядро

$$
K(t)=\omega_{1}^{2} \operatorname{Exp}(2, \alpha ; \lambda ; t)+\omega_{2}^{2} t
$$

можно показать, что

$$
\left(\frac{d}{d t}\right)^{2} K(t)=\lambda \operatorname{Exp}(\alpha, \alpha ; \lambda ; t)=\lambda t^{\alpha-1} E_{\alpha}\left(\lambda t^{\alpha} ; \alpha\right)
$$

и имеет при $\alpha \in(0,1)$ суммируемую особенность в нуле, причём

$$
I_{0 t}^{1-\alpha} \operatorname{Exp}(\alpha, \alpha ; \lambda ; t)=\operatorname{Exp}(\alpha, 1 ; \lambda ; t) \in A C[0, T]
$$

и $\lim _{t \rightarrow 0+} \operatorname{Exp}(\alpha, 1 ; \lambda ; t)=1$. 
Из вышеизложенного следует, что решение интегрального уравнения (14) существует, единственно и может быть найдено методом последовательных приближений в требуемом для корректности задачи типа Коши (6), (9), (13) классе функций.

Для применения метода последовательных приближений удобно привести уравнение (12) к интегральному уравнению с ядром в другой записи. Для этого рассмотрим уравнение (12) как обыкновенное дифференциальное уравнение второго порядка с правой частью

$$
F(t)=f_{1}(t)+\frac{u_{0}}{m} \operatorname{Exp}(\alpha, \alpha ; \lambda ; t)-\omega_{1}^{2} \lambda E_{0 t ; \lambda}^{\alpha, \alpha} x .
$$

Его решение с начальными условиями (13) будет иметь вид

$$
x(t)=x_{0} \cos \omega_{0} t+\frac{\dot{x_{0}}}{\omega_{0}} \sin \omega_{0} t+\frac{1}{\omega_{0}} \int_{0}^{t} \sin \omega_{0}(t-\tau) F(\tau) d \tau .
$$

Вычислим интеграл

$$
\begin{aligned}
I(t)=\int_{0}^{t} \sin \omega_{0}(t-\tau) \operatorname{Exp}(\alpha, \alpha ; \lambda ; \tau) d \tau & =\sin \omega_{0}(t-\tau) * \operatorname{Exp}(\alpha, \alpha ; \lambda ; t)= \\
& =\int_{0}^{t} \operatorname{Exp}(\alpha, \alpha ; \lambda ; t-\tau) \sin \omega_{0} \tau d \tau
\end{aligned}
$$

Используем запись оператора $E_{0 t ; \lambda}^{\alpha, \sigma}(10)$ и представление $\sin \omega_{0} t$ в терминах обобщённой дробной экспоненциальной функции:

$$
\begin{gathered}
E_{0 t ; \lambda}^{\alpha, \sigma} f=\int_{0}^{t} \operatorname{Exp}(\sigma, \alpha ; \lambda ; t-\tau) f(\tau) d \tau \\
\sin \omega_{0} t=\omega_{0} t E_{2}\left(-\omega_{0}^{2} t^{2} ; 2\right)=\operatorname{Exp}\left(2,2 ;-\omega_{0}^{2} ; t\right) .
\end{gathered}
$$

Затем воспользуемся формулой [8]

$$
E_{0 t ; \lambda_{1}}^{\mu, \sigma} \operatorname{Exp}\left(\beta, \nu ; \lambda_{2} ; t\right)=\operatorname{Exp}\left(\alpha, \beta, \mu+\nu ; \lambda_{1}, \lambda_{2} ; t\right),
$$

где возникает обобщённая дробная экспоненциальная функция

$$
\operatorname{Exp}\left(\alpha, \beta, \mu ; \lambda_{1}, \lambda_{2} ; t\right)=t^{\mu-1} E_{\alpha, \beta}\left(\lambda_{1} t^{\alpha}, \lambda_{2} t^{\alpha} ; \mu\right),
$$

порожденная трехпараметрической функцией типа Миттаг-Леффлера двух аргументов:

$$
E_{\alpha, \beta}(x, y ; \mu)=\sum_{k, n=0}^{\infty} \frac{x^{k} y^{n}}{\Gamma(\alpha k+\beta n+\mu)}
$$

Тогда с учётом формул (16)-(18) имеем

$$
I(t)=E_{0 t ; \lambda}^{\alpha, \alpha} \sin \omega_{0} t=\omega_{0} E_{0 t ; \lambda}^{\alpha, \alpha} \operatorname{Exp}\left(2,2 ;-\omega_{0}^{2} ; t\right)=\omega_{0} \operatorname{Exp}\left(\alpha, 2 ; \alpha+2 ; \lambda,-\omega_{0}^{2} ; t\right) .
$$

Известно, что

$$
x E_{\alpha, \beta}(x, y ; \mu+\alpha)=E_{\alpha, \beta}(x, y ; \mu)-E_{\beta}(y ; \mu) .
$$


Окончательно, используя (19) и (20), найдём

$$
\begin{gathered}
I(t)=\omega_{0} \operatorname{Exp}\left(\alpha, 2 ; \alpha+2 ; \lambda,-\omega_{0}^{2} t\right)=\omega_{0} t^{\alpha+1} E_{\alpha, 2}\left(\lambda t^{2},-\omega_{0}^{2} t^{2} ; \alpha+2\right)= \\
=\frac{\omega_{0}}{\lambda} t\left(\lambda t^{\alpha}\right) E_{\alpha, 2}\left(\lambda t^{\alpha},-\omega_{0}^{2} t^{2} ; \alpha+2\right)= \\
=\frac{\omega_{0}}{\lambda} t\left[E_{\alpha, 2}\left(\lambda t^{\alpha},-\omega_{0}^{2} t^{2} ; 2\right)-E_{2}\left(-\omega_{0}^{2} t^{2} ; 2\right)\right]= \\
=\frac{1}{\lambda}\left[\omega_{0} \operatorname{Exp}\left(\alpha, 2, \alpha ; \lambda,-\omega_{0}^{2} ; t\right)-\sin \omega_{0} t\right] .
\end{gathered}
$$

Используя результат вычислений в (21), запишем уравнение (15) в виде интегрального уравнения

$$
x(t)+\lambda \frac{\omega_{1}^{2}}{\omega_{0}} \int_{0}^{t} \sin \omega_{0}(t-\tau)\left(E_{0 \tau ; \lambda}^{\alpha, \alpha} x\right)(\tau) d \tau=\varphi(t)
$$

где

$$
\varphi(t)=x_{0} \cos \omega_{0} t+\frac{1}{\omega_{0}}\left(\dot{x}_{0}-\frac{u_{0}}{\lambda m}\right) \sin \omega_{0} t+\frac{u_{0}}{\lambda m} \operatorname{Exp}\left(\alpha, 2, \alpha ; \lambda,-\omega_{0}^{2} ; t\right) .
$$

\section{Обозначим}

$$
\left(A_{0 t}^{\alpha} x\right)(t)=\frac{1}{\omega_{0}} \int_{0}^{t} \sin \omega_{0}(t-\tau)\left(E_{0 \tau ; \lambda}^{\alpha, \alpha} x\right)(\tau) d \tau
$$

- интегральный оператор, возникший в уравнении (22), и найдём его ядро в явном виде.

Запишем в (23) оператор $E_{0 \tau ; \lambda}^{\alpha, \alpha} x$ по определению $(16)$, изменим порядок интегрирования и выполним замену переменной интегрирования во внутреннем интеграле по формуле $\tau-s=(t-s) z$. Получим

$$
\begin{aligned}
& A_{0 t}^{\alpha} x=\frac{1}{\omega_{0}} \int_{0}^{t} \sin \omega_{0}(t-\tau) \int_{0}^{\tau} \operatorname{Exp}(\alpha, \alpha ; \lambda ; \tau-s) x(s) d s= \\
& =\frac{1}{\omega_{0}} \int_{0}^{t} x(s) d s \int_{s}^{t} \operatorname{Exp}(\alpha, \alpha ; \lambda ; \tau-s) \sin \omega_{0}(t-\tau) d \tau= \\
& =\frac{1}{\omega_{0}} \int_{0}^{t}(t-s) x(s) d s \int_{0}^{1} \operatorname{Exp}\left[\alpha, \alpha ; \lambda ;(\tau-s)^{\alpha} z^{\alpha}\right] \sin \omega_{0}[(t-s)(1-z)] d z= \\
& =\frac{1}{\omega_{0}} \int_{0}^{t}(t-s)^{\alpha} x(s) d s \int_{0}^{1} z^{\alpha-1} E_{\alpha}\left[\lambda(t-s)^{\alpha} z^{\alpha} ; \alpha\right] \sin \omega_{0}[(t-s)(1-z)] d z .
\end{aligned}
$$

Записывая подынтегральные функции во внутреннем интеграле (24) по определению в форме рядов и выполняя почленное интегрирование их произведения, в конечном итоге можем показать, что

$$
\begin{aligned}
\int_{0}^{1} z^{\alpha-1} E_{\alpha}\left[\lambda(t-s)^{\alpha} z^{\alpha} ; \alpha\right] \sin \omega_{0}[(t-s) & (1-z)] d z= \\
& =E_{\alpha}\left[\lambda(t-s)^{\alpha} ; \alpha+2\right] \sin \omega_{0}(t-s) .
\end{aligned}
$$


Таким образом, интегральное уравнение (22), к которому редуцируется начальная задача типа Коши с условиями (8) и (13) для дифференциального уравнения (6), может быть записано в виде

$$
x(t)+\lambda \omega_{1}^{2}\left(A_{0 t}^{\alpha} x\right)(t)=\varphi(t),
$$

где оператор

$$
A_{0 t}^{\alpha} x=\int_{0}^{t}(t-s)^{\alpha} E_{\alpha}\left[\lambda(t-s)^{\alpha} ; \alpha+2\right] \sin \omega_{0}(t-s) x(s) d s
$$

имеет непрерывное ядро и, следовательно, интегральное уравнение (25) безусловно разрешимо. Как и в первом случае, его решение можно найти методом последовательных приближений.

\section{БИБЛИОГРАФИЧЕСКИЙ СПИСОК}

1. С. Г. Самко, А. А. Килбас, О. И. Маричев, Интегралы и производные дробного порядка и некоторые их приложения. Минск: Наука и техника, 1987. 688 с. [S. G. Samko, A. A. Kilbas, O. I. Marichev, Integrals and derivatives of fractional order and some of their applications. Minsk: Nauka i Tekhnika, 1987. 688 pp.]

2. А. М. Нахушев, Дробное исчисление и его применение. М.: Физматлит, 2003. 271 с. [A. M. Nakhushev, Fractional calculus and its applications. Moscow: Fizmatlit, 2003. 271 pp.]

3. Е. Н. Огородников, "Математические модели дробных осцилляторов, постановка и структура решения задачи Коши" / В сб.: Трудь шестой Всероссийской научной конферениии с международным участием (1-4 июня 2009 г.). Часть 1: Математические модели механики, прочности и надёжности элементов конструкций/ Матем. моделирование и краев. задачи. Самара: СамГТУ, 2009. С. 177-181. [E. N. Ogorodnikov, "Mathematical models of the fractional oscillator, setting and structure of the Cauchy problem" / In: Proceedings of the Sixth All-Russian Scientific Conference with international participation (1-4 June 2009). Part 1 / Matem. Mod. Kraev. Zadachi. Samara: SamGTU, 2009. Pp. 177-181].

4. F. Mainardi, "Fractional relaxation-oscillation and fractional diffusion-wave phenomena" // Chaos, Solitons and Fractals, 1996. Vol. 7, no. 9. Pp. 1461-1477.

5. A. A. Kilbas, H. M. Srivastava, J. J. Trujillo, Theory and Applications of Fractional Differential Equations / North-Holland Mathematics Studies. Vol. 204 / ed. J. van Mill. Amsterdam: Elsevier, 2006. 523 pp.

6. Е. Н. Огородников, Н. С. Яшагин, "Некоторые специальные функции в решении задачи Коши для одного дробного осцилляционного уравнения" // Bестн. Сам. гос. техн. ун-та. Сер. Физ.-мат. науки, 2009. №1(18). С. 276-279. [E. N. Ogorodnikov, N. S. Yashagin, "Some special functions in the solution to Cauchy problem for a fractional oscillating equation"// Vestn. Samar. Gos. Tekhn. Univ. Ser. Fiz.-Mat. Nauki, 2009. no. 1(18). Pp. 276-279].

7. Е. Н. Огородников, Н. С. Яшагин, "Постановка и решение задач типа Коши для дифференциальных уравнений второго порядка с дробными производными Римана-Лиувилля" // Вестн. Сам. гос. техн. ун-та. Сер. Физ.-мат. науки, 2010. №1(20). С. 24-36. [E. N. Ogorodnikov, N. S. Yashagin, "Setting and solving of the Cauchy type problems for the second order differential equations with Riemann-Liouville fractional derivatives" // Vestn. Samar. Gos. Tekhn. Univ. Ser. Fiz.-Mat. Nauki, 2010. no. 1(20). Pp. 24-36].

8. Е. Н. Огородников, Н. С. Яшагин, В. П Радченко, "Реологические модели вязкоупругого тела с памятью и дифференциальные уравнения дробных осцилляторов" // Вестн. Сам. гос. техн. ун-та. Сер. Физ.-мат. науки, 2011. №1(22). С. 255-268. 
[E. N. Ogorodnikov, N. S. Yashagin, V. P. Radchenko, "Rheological model of viscoelastic body with memory and differential equations of fractional oscillator" // Vestn. Samar. Gos. Tekhn. Univ. Ser. Fiz.-Mat. Nauki, 2011. no.1(22). Pp. 255-268].

9. M. Caputo, F. Mainardi, "A new dissipation model based on memory mechanism" // Pure Appl. Geophys., 1971. Vol. 91, no. 1. Pp. 134-147.

10. R. L. Bagley, P. J. Torvik, "On the Fractional Calculus Model of Viscoelastic Behavior" // J. Rheol., 1986. Vol. 30, no. 1. Pp. 133-155.

11. Ю. Н. Работнов, Элементы наследственной механики твёрдых тел. М.: Наука, 1977. 383 c. [Yu. N. Rabotnov, Elements of continuum mechanics of materials with memory. Moscow: Nauka, 1977. 383 pp.]

12. I. H. Barrett, "Differential equations of non-integer orde" // Canad. J. Math., 1954. Vol.6, no. 4. Pp. 529-541.

13. Е. Н. Огородников, "Некоторые аспекты теории начальных задач для дифференциальных уравнений с производными Римана-Лиувилля"// Вестн. Сам. гос. техн. ун-та. Сер. Физ.-мат. науки, 2010. №5(21). С. 10-23. [E. N. Ogorodnikov, "Some aspects of initial value problems theory for differential equations with Riemann-Liouville derivatives"// Vestn. Samar. Gos. Tekhn. Univ. Ser. Fiz.-Mat. Nauki, 2010. no. 5(21). Pp. 10-23].

Поступила в редакцию 27/I/2013;

в окончательном варианте - 17/III/2013.

MSC: 34A08; 26A33, 45K05

\section{ON A CLASS OF FRACTIONAL DIFFERENTIAL EQUATIONS FOR MATHEMATICAL MODELS OF DYNAMIC SYSTEMS WITH MEMORY}

\section{E. N. Ogorodnikov}

Samara State Technical University,

244, Molodogvardeyskaya st., Samara, 443100, Russia.

E-mail: eugen.ogo@gmail.com

The differential equation with Riemann-Liouville fractional derivatives is considered. The equations of this class are proposed as a model fractional oscillating equations for the description, analysis and investigation of oscillatory processes in dynamic systems with memory. Such a kind of equations obtainment is based on the hypothesis supposed the existence of the non-ideal viscoelastic connection in the one-dimensional dynamic system, which is associated with the fractional analogy of Zener rheologic model of the viscoelastic body. It's shown, that the initial values problems with Cauchy type conditions can be reduced equivalently to the Volterra type integral equations with the differentiable kernels. This circumstance allow to use the method of successive approximation to resolve these integral equations. It's indicated, that such a kind of differential equations can be interesting as mathematical models of nonlinear dynamic systems behavior.

Key words: differential and integral equations with fractional Riemann-Liouville operators, fractional oscillators, fractional oscillating equations, rheological model of viscoelastic body with memory, Mittag-Leffler type special functions, Volterra type integral equations with special functions in kernel.

Original article submitted 27/I/2013;

revision submitted $17 / \mathrm{III} / 2013$.

Eugeniy N. Ogorodnikov (Ph. D. (Phys. \& Math.)), Associate Professor, Dept. of Applied Mathematics \& Computer Science. 